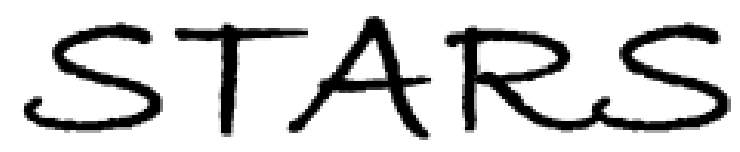

University of Central Florida

STARS

Faculty Bibliography 2000s

Faculty Bibliography

$1-1-2009$

\title{
Low voltage blue-phase liquid crystal displays
}

\author{
Linghui Rao \\ University of Central Florida \\ Zhibing Ge \\ University of Central Florida \\ Shin-Tson Wu \\ University of Central Florida \\ Seung Hee Lee
}

Find similar works at: https://stars.library.ucf.edu/facultybib2000 University of Central Florida Libraries http://library.ucf.edu

This Article is brought to you for free and open access by the Faculty Bibliography at STARS. It has been accepted for inclusion in Faculty Bibliography 2000 s by an authorized administrator of STARS. For more information, please contactSTARS@ucf.edu.

\section{Recommended Citation}

Rao, Linghui; Ge, Zhibing; Wu, Shin-Tson; and Lee, Seung Hee, "Low voltage blue-phase liquid crystal displays" (2009). Faculty Bibliography 2000s. 2036.

https://stars.library.ucf.edu/facultybib2000/2036

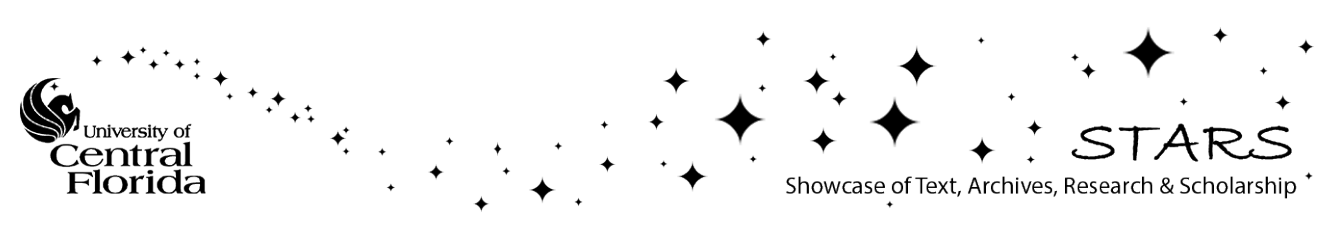




\section{Low voltage blue-phase liquid crystal displays}

Cite as: Appl. Phys. Lett. 95, 231101 (2009); https://doi.org/10.1063/1.3271771

Submitted: 04 November 2009 . Accepted: 13 November 2009 . Published Online: 07 December 2009

Linghui Rao, Zhibing Ge, Shin-Tson Wu, and Seung Hee Lee

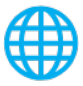

\section{ARTICLES YOU MAY BE INTERESTED IN}

Electro-optics of polymer-stabilized blue phase liquid crystal displays

Applied Physics Letters 94, 101104 (2009); https://doi.org/10.1063/1.3097355

Extended Kerr effect of polymer-stabilized blue-phase liquid crystals

Applied Physics Letters 96, 071105 (2010); https://doi.org/10.1063/1.3318288

A large Kerr constant polymer-stabilized blue phase liquid crystal

Applied Physics Letters 98, 081109 (2011); https://doi.org/10.1063/1.3559614

\section{Applied Physics Letters}

Mid-IR and THz frequency combs special collection

Read Now! 


\title{
Low voltage blue-phase liquid crystal displays
}

\author{
Linghui Rao, ${ }^{1}$ Zhibing Ge, ${ }^{1}$ Shin-Tson Wu, ${ }^{1, a)}$ and Seung Hee Lee ${ }^{2}$ \\ ${ }^{1}$ College of Optics and Photonics, University of Central Florida, Orlando, Florida 32816, USA \\ ${ }^{2}$ Department of Polymer-Nano Science and Engineering, Chonbuk National University, Chonju, \\ Chonbuk 561-756, Republic of Korea
}

(Received 4 November 2009; accepted 13 November 2009; published online 7 December 2009)

\begin{abstract}
A protrusion electrode structure is proposed to dramatically lower the operation voltage of the emerging blue-phase liquid crystal displays (BP-LCDs). Simulation results indicate that the generated horizontal electric field is not only strong but also penetrates deeply into the bulk LC layer. As a result, a low voltage $\left(\sim 10 \mathrm{~V}_{\text {rms }}\right)$ and reasonably high transmittance $(\sim 70 \%)$ BP-LCD can be achieved. This approach enables the BP-LCDs to be addressed by amorphous silicon thin-film transistors (TFTs). Widespread application of TFT BP-LCDs is foreseeable. (C) 2009 American Institute of Physics. [doi:10.1063/1.3271771]
\end{abstract}

Kerr-effect-induced isotropic-to-anisotropic transition in blue-phase liquid crystal (BPLC) ${ }^{1-6}$ has potential to become next-generation display technology because it exhibits following four revolutionary features. (1) Its response time is in submillisecond range which is approximately ten times faster than the present nematic LC displays (LCDs). Thus, it enables color-sequential display with a red, green, and blue light-emitting diode backlight so that the conventional pigment color filters can be eliminated. The removal of color filters leads to approximately three times higher optical efficiency, three times higher resolution, and wider color gamut (>120\% NTSC). (2) It does not require alignment layer so that the fabrication process is greatly simplified. (3) The voltage-off state is optically isotropic and voltage-on state forms multi-domain structures so that its viewing angle is wide and symmetric. (4) Its transmittance is insensitive to the cell gap as long as the cell gap exceeds $\sim 2-3 \mu \mathrm{m}$, $^{7,8}$ This feature is particularly desirable for fabricating largescreen LCDs, in which cell-gap uniformity is a big concern. However, two major technical challenges: high operating voltage and comparatively low optical efficiency remain to be overcome before widespread applications can take off.

Current research of BP LCDs employs traditional interdigital strip electrode, known as in-plane switching (IPS) cell. ${ }^{9-11}$ The on-state voltage $\left(\mathrm{V}_{\text {on }}\right)$ where peak transmittance occurs is $\sim 50 \mathrm{~V}_{\text {rms }}$ with a Kerr constant $K \sim 10 \mathrm{~nm} / \mathrm{V}^{2}{ }^{12}$ There is an urgent need to reduce $\mathrm{V}_{\text {on }}$ to $\sim 10 \mathrm{~V}_{\text {rms }}$ in order to drive the devices with mainstream amorphous-silicon thinfilm transistors (a-Si TFTs).

In this letter, we propose a protrusion electrode structure to produce strong horizontal electric field and, moreover, this field penetrates deeply into the LC layer. By optimizing the electrode configuration, we have successfully lowered the operating voltage to $\sim 10 \mathrm{~V}_{\text {rms }}$ while keeping a relatively high $(\sim 70 \%)$ optical efficiency.

When a Kerr medium with centrosymmetric point groups is subject to an external electric field, the induced birefringence $(\delta n)$ can be expressed as ${ }^{13}$

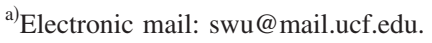

$$
\delta n=\lambda K E^{2} .
$$

Here, $\lambda$ is the wavelength, $K$ is the Kerr constant, and $E$ is the amplitude of the electric field. Equation (1) is valid only in the low electric field regime while the induced refractive index change does not exceed the intrinsic effective birefringence of the LC composite. That is, $\delta n$ will saturate as the electric field reaches a saturation value. ${ }^{7}$ Recently, this Kerr effect is also observed in polymer-stabilized blue-phase LC media which exhibit a wide temperature blue-phase. ${ }^{4-6}$ From Eq. (1), the LC system is optically isotropic at $E=0$, but becomes anisotropic as $E$ increases. Therefore, we often refer to this Kerr-effect-induced birefringence as isotropic-toanisotropic transition.

In the IPS blue-phase LC structure, when an electric field is applied the molecular reorientations take place in the double-twisted cylinders. ${ }^{14}$ From macroscopic viewpoint, the effective optic axis of the induced refractive index anisotropy is along the electric field vector. Only the horizontal electric field contributes to the transmittance of the BPLC cell. Therefore, to enhance the transmittance and lower the onstate voltage, it is essential to create a strong horizontal field and make it penetrating deeper into the LC layer.

In the conventional planar IPS structure, we could strengthen the horizontal electric field by reducing the electrode gap. However, as the electrode gap gets narrower the penetration depth becomes shallower which leads to a reduced optical efficiency. To overcome this problem, we propose a protrusion electrode structure, as depicted in Fig. 1. The BPLC cell is sandwiched between two crossed polarizers, and the pixel and common protrusion electrodes have trapezoid shape. Their dimension is defined as follows: $w_{1}$ is the bottom width, $w_{2}$ is the top width, $h$ is the height, and $l$ is the space between common and pixel electrodes. In this design, strong electric fields are generated between the pixel and common electrodes ( $x$-axis), and more importantly, the field is able to penetrate deeply into the LC bulk region (z-axis). The former plays a key role to reduce operating voltage while the latter helps to achieve high transmittance.

The protrusion structures have been widely used in multidomain vertical alignment LCDs for obtaining wide viewing angle. ${ }^{15,16}$ Therefore, the fabrication of protrusion electrodes (by coating indium tin oxide on top of protrusions) we propose here is not a technical hurdle. A typical protrusion 


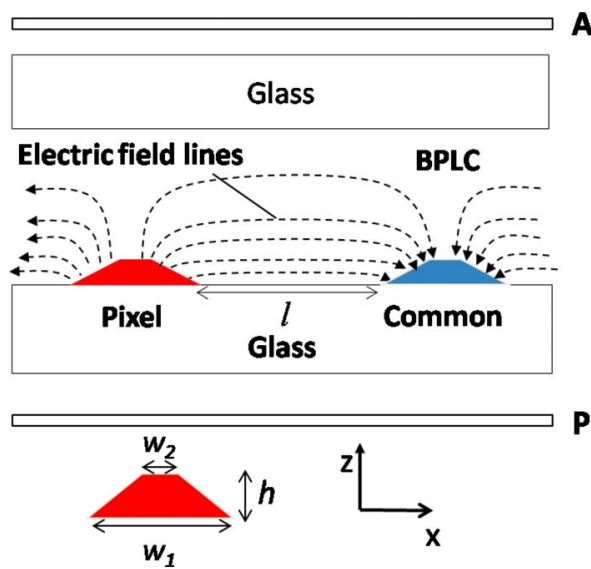

FIG. 1. (Color online) Schematic structure of the blue-phase LCD with protrusion electrodes: A and $\mathrm{P}$ denote the analyzer and polarizer.

height is $\sim 2 \mu \mathrm{m}$; moreover, it can be made transparent with organic positive photoresist so the aperture ratio of the LC panel will not be sacrificed. For the protrusion electrode with $w_{1}=2 \mu \mathrm{m}, w_{2}=1 \mu \mathrm{m}, h=2 \mu \mathrm{m}$, and $l=4 \mu \mathrm{m}$ shown in Fig. 2, the peak transmittance is $\sim 71 \%$ (normalized to the transmittance of two parallel polarizers) at $17 \mathrm{~V}_{\text {rms }}$. Compared to the conventional IPS structure with electrode width $w=2 \mu \mathrm{m}$ and electrode gap $l=4 \mu \mathrm{m}$ (designated as IPS $2-4$ ) whose $\mathrm{V}_{\text {on }} \sim 38 \mathrm{~V}_{\text {rms }}$ and peak transmittance $\sim 66.5 \%$, our protrusion electrode shows a significant reduction in driving voltage while maintaining a relatively high transmittance.

To understand the underlying physics, we plot in Figs. $3(\mathrm{a})$ and 3(b) the effective induced-birefringence $\left(\delta n_{\text {eff }}\right)$ distribution for the IPS 2-4 and protrusion electrode structures. Here, $\delta n_{\text {eff }}$ is the effective birefringence that the normal incident light experiences. It is different from the induced birefringence $\delta n$ in Eq. (1), which is the overall induced birefringence of the BPLC material regardless of the electric field direction and the effectiveness whether the $\delta n$ will contribute to the transmittance. From Fig. 3, $\delta n_{\text {eff }}$ is almost zero on top of the electrode for both structures since the electric field there is almost vertically distributed so that it does not cause phase retardation to the normal incident light. In Fig. 3(b), the electric fields penetrate deeper into the LC layer and produce a larger $\delta n_{\text {eff }}$ in the effective region. This is the

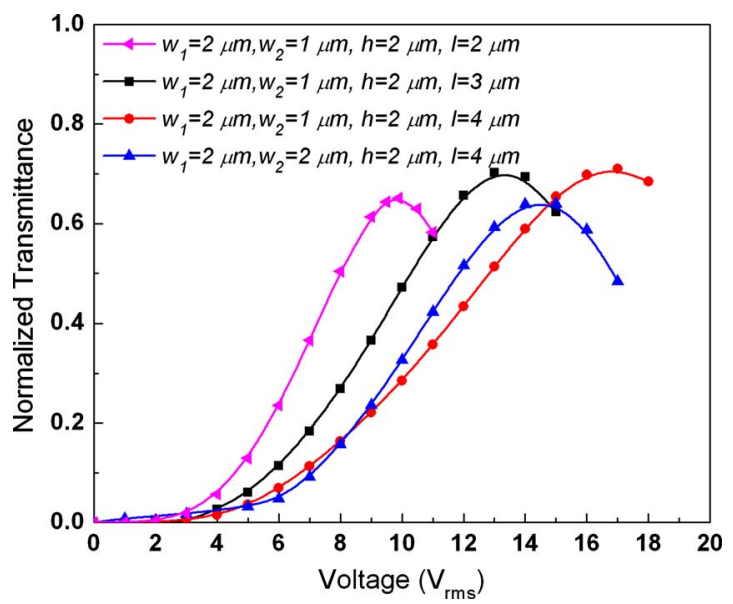

FIG. 2. (Color online) Normalized VT curves for protrusion electrodes with different dimensions.

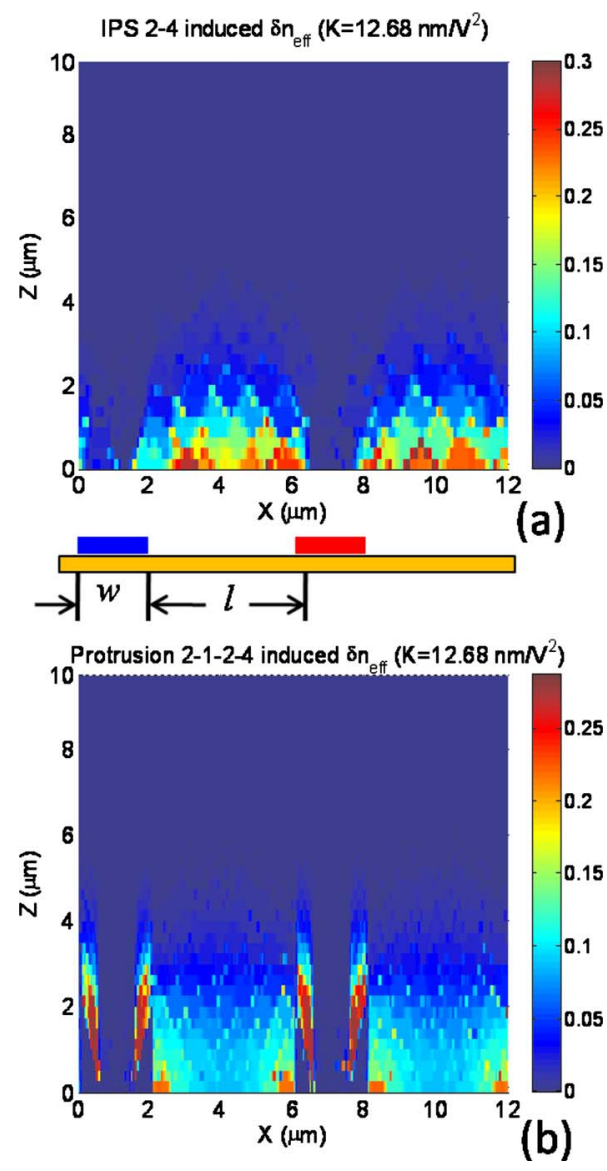

FIG. 3. (Color online) Induced effective birefringence $\left(\delta n_{\mathrm{eff}}\right)$ distribution for (a) conventional IPS cell with strip electrode with electrode width $w=2 \mu \mathrm{m}$, spacing $l=4 \mu \mathrm{m}$, and cell gap $\mathrm{d}=10 \mu \mathrm{m}$, and (b) protrusion electrode with $w_{1}=2 \mu \mathrm{m}, w_{2}=1 \mu \mathrm{m}, h=2 \mu \mathrm{m}$, and $l=4 \mu \mathrm{m}$, cell gap $\mathrm{d}=10 \mu \mathrm{m}$

reason why a much lower on-state voltage is needed for the protrusion electrode design. In addition, the induced birefringence at the spacing area decays fast with the cell gap. Thus, as long as the cell gap is larger than the effective penetrating depth in the vertical direction, the transmittance will be insensitive to the cell gap. Although for both structures, the vertically distributed electric field does not contribute to the transmittance of the normal incident light, it will compensate the off-angle incident light. The increasingly curved electric field in $z$-direction of protrusion electrode compensates more than the strip electrodes due to its deeper penetration layer into the LC bulk region. If we compare the isobrightness contour plots (figures not shown here), the polar angle of the protrusion structure with a transmittance of $\sim 60 \%$ is over $50^{\circ}$ while the strip electrode structure is only $\sim 30^{\circ}$.

Shown in Fig. 2 is the influence of electrode dimension on the voltage-dependent transmittance (VT) curve. In comparison to the structure with parameters: $w_{1}=2 \mu \mathrm{m}, w_{2}$ $=1 \mu \mathrm{m}, h=2 \mu \mathrm{m}$, and $l=4 \mu \mathrm{m}$, if we decrease the spacing width $l$ to 3 or $2 \mu \mathrm{m}$, then the on-state voltage is lowered to 13 or $9.9 \mathrm{~V}_{\mathrm{rms}}$, respectively. This is attributed to the stronger electric field generated as the electrode gap gets smaller. But the tradeoff is the lower transmittance, because the effective volume with horizontal electric field that accumulates the phase for the transmittance is smaller. If the size of $w_{2}$ is increased to $2 \mu \mathrm{m}$, the electric field near the bottom of the cell will be more uniformly distributed in horizontal direc- 


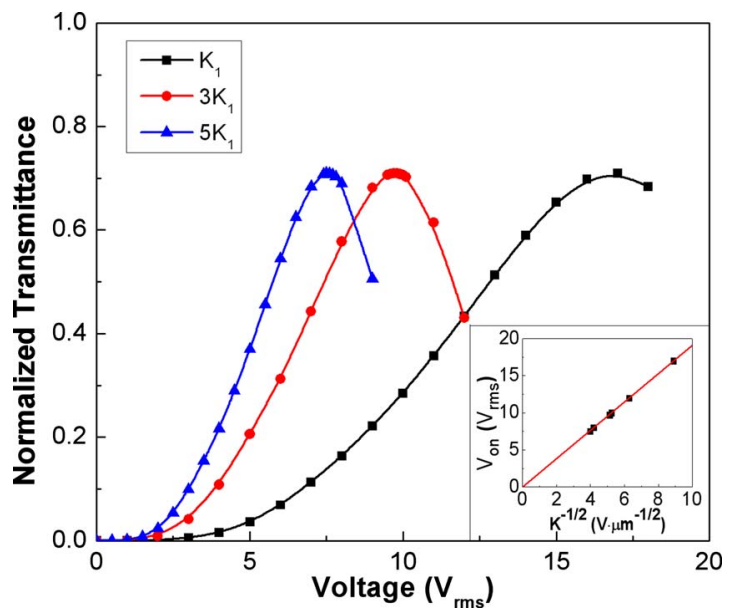

FIG. 4. (Color online) VT curves with different Kerr constants for protrusion electrode structure with $w_{1}=2 \mu \mathrm{m}, w_{2}=1 \mu \mathrm{m}, h=2 \mu \mathrm{m}$, and $l=4 \mu \mathrm{m}$, cell gap $\mathrm{d}=10 \mu \mathrm{m}$, inset is the linear plot between on-state voltage and $1 / \sqrt{K}$.

tion. As a result, denoted by the blue line in Fig. 2, the on-state voltage occurs at $\sim 14.5 \mathrm{~V}_{\text {rms }}$, but its transmittance is somewhat lower. Again, this is because a larger $w_{2}$ reduces the effective spacing area between the electrodes. In this way, a larger taper angle will have a lower driving voltage but also a lower transmittance. If we increase the protrusion height $h$ to $4 \mu \mathrm{m}$, the peak transmittance (results not shown) would increase to $74.2 \%$ at $13 \mathrm{~V}_{\mathrm{rms}}$. The electric field in this case is stronger and more uniform as compared to the one with a $2 \mu \mathrm{m}$ height, thus a lower driving voltage is needed. Meanwhile, the penetration of electric field gets deeper into the LC medium which enhances the transmittance. However, a $4 \mu \mathrm{m}$ height protrusion electrode may be difficult to fabricate.

In the voltage-off state, blue-phase LC is optically isotropic so the dark state is very good. We have calculated the isocontrast contours for the protrusion electrode structure with dimensions of $\mathrm{d}=10 \mu \mathrm{m}, w_{1}=2 \mu \mathrm{m}, w_{2}=1 \mu \mathrm{m}, h$ $=2 \mu \mathrm{m}$, and $l=4 \mu \mathrm{m}$ and $\lambda=550 \mathrm{~nm}$. The contrast ratio (CR) of $200: 1$ is over $30^{\circ}$ without any compensation film. Adding a half-wave biaxial film ${ }^{7}$ greatly suppresses the offaxis light leakage of the two crossed polarizers, so that $\mathrm{CR}$ $=1000: 1$ can reach about $60^{\circ}$. This result is comparable to that of the strip-electrode IPS BPLC cell we reported previously. ${ }^{7}$ While blue phase LCs have a really good dark state, the contrast ratio is mainly governed by the on-state transmittance.

In addition to optimizing the device structure for generating strong and deep electric fields, it is equally important to develop LC materials with a larger Kerr constant. Figure 4 depicts the simulated VT curves at $\lambda=550 \mathrm{~nm}$ under different Kerr constants $\left(K_{1}=12.68 \mathrm{~nm} / \mathrm{V}^{2}, K_{2}=3 K_{1}\right.$, and $K_{3}=5 K_{1}$ ) for the protrusion electrode structure with dimensions of $\mathrm{d}=10 \mu \mathrm{m}, w_{1}=2 \mu \mathrm{m}, w_{2}=1 \mu \mathrm{m}, h=2 \mu \mathrm{m}$, and $l=4 \mu \mathrm{m}$. With the increased Kerr constant, the on-state voltage is reduced from 17 to 9.7 and $7.5 \mathrm{~V}_{\mathrm{rms}}$. The low voltage operation would reduce the power consumption of the display devices. Amazingly, the on-state voltage is linearly proportional to $K^{-1 / 2}$, although the electric field generated from the protrusion electrodes is not uniform. Shown in the inset of Fig. 4, the red line is the linear fitting for $K_{1}, K_{2}$, $K_{3}, K_{4}=2 K_{1}$, and $K_{5}=4.5 K_{1}$ with the following equation:

$$
\mathrm{V}_{\text {on }}=A \cdot \frac{1}{\sqrt{K}} \text {. }
$$

Here, the proportionality constant $A=1.91 \mu \mathrm{m}^{1 / 2}$. For a given structure, the distribution of induced birefringence in an on-state voltage will remain the same even the $K$ values are different. According to Eq. (1), if the electric field $E$ is uniform in the cell, $K E^{2}$ will be a constant. As $E=V / L, K V^{2}$ is also a constant, and Eq. (2) holds for this straightforward case. In the structure with protrusion electrodes, the $K E^{2}$ value may differ at different area because of the $E$ distribution, but it remains a constant for the same area in the cell despite of different $K$ value. The voltage $V$ is related to the electric field $E$ as $E=(\partial V) /(\partial X)$ ( $\partial X$ is the infinitesimal change in distance), as long as the cell is operated under the on-state voltage, the $K V^{2}$ value would stay constant for the same area of the cell regardless of the $K$ variation. Moreover, the unchanged peak transmittance in Fig. 4 for different $K$ values also results from the same induced birefringence distribution. Or, we can conclude that for a given structure, while Kerr constant $K$ represents the influence from the material side to the driving voltage, the constant $A$ represents the effect from the device design.

In conclusion, we have proposed a feasible protrusion electrode structure for dramatically reducing the operating voltage of blue-phase LCDs while keeping a reasonably high transmittance. By optimizing the protrusion structure, we have successfully reduced the operating voltage to $\sim 10 \mathrm{~V}_{\mathrm{rms}}$. This is an important milestone because the device can be driven by a-Si TFTs. With continuous development of large- $K$ LC materials, the voltage can be reduced further and widespread applications of blue-phase LCDs is foreseeable.

The authors are indebted to Chi-Mei Optoelectronics for partial financial support, and S. Gauza and K. M. Chen for stimulating discussions.

${ }^{1}$ S. Meiboom, J. P. Sethna, W. P. Anderson, and W. F. Brinkman, Phys. Rev. Lett. 46, 1216 (1981).

${ }^{2}$ P. R. Gerber, Mol. Cryst. Liq. Cryst 116, 197 (1985).

${ }^{3}$ H. F. Gleeson and H. J. Coles, Liq. Cryst. 5, 917 (1989).

${ }^{4}$ H. Kikuchi, M. Yokota, Y. Hiskado, H. Yang, and T. Kajiyama, Nature Mater. 1, 64 (2002).

${ }^{5}$ Y. Hisakado, H. Kikuchi, T. Nagamura, and T. Kajiyama, Adv. Mater. 17, 96 (2005).

${ }^{6}$ Y. Haseba, H. Kikuchi, T. Nagamura, and T. Kajiyama, Adv. Mater. 17, 2311 (2005).

${ }^{7}$ Z. Ge, S. Gauza, M. Jiao, H. Xianyu, and S. T. Wu, Appl. Phys. Lett. 94, 101104 (2009).

${ }^{8}$ Z. Ge, L. Rao, S. Gauza, and S. T. Wu, J. Disp. Technol 5, 250 (2009).

${ }^{9}$ M. Oh-e and K. Kondo, Appl. Phys. Lett. 67, 3895 (1995).

${ }^{10}$ R. A. Soref, J. Appl. Phys. 45, 5466 (1974).

${ }^{11}$ W. Helfrich and M. Schadt, Phys. Rev. Lett. 27, 561 (1971).

${ }^{12}$ H. Kikuchi, Y. Haseba, S. Yamamoto, T. Iwata, and H. Higuchi, SID Int Symp. Digest Tech. Papers 39, 578 (2009).

${ }^{13}$ A. Yariv and P. Yeh, Optical Waves in Crystals (Wiley, New York, 1984)

${ }^{14}$ P. P. Crooker, in Chirality in Liquid Crystals, edited by H. S. Kitzerow and C. Bahr (Springer, New York, 2001), Chap. 7.

${ }^{15}$ A. Takeda, S. Kataoka, T. Sasaki, H. Chida, H. Tsuda, K. Ohmuro, T. Sasabayashi, Y. Koike, and K. Okamoto, SID Int. Symp. Digest Tech. Papers 29, 1077 (1998).

${ }^{16}$ J. O. Kwag, K. C. Shin, J. S. Kim, S. G. Kim, and S. S. Kim, SID Int. Symp. Digest Tech. Papers 31, 256 (2000). 